\title{
Cost-benefit Analysis on Introduction of Macrobrachium rosenbergii (De Man, 1879) in Puthumurippu Medium Perennial Reservoir in Sri Lanka
}

\author{
R. Rajeevan*, U. Edirisinghe ${ }^{1}$ and A.R.S.B. Athauda ${ }^{1}$ \\ Postgraduate Institute of Agriculture \\ University of Peradeniya \\ Sri Lanka
}

\begin{abstract}
Macrobrachium rosenbergii, a giant freshwater prawn, was newly introduced to Puthumurippu medium perennial reservoir in Kilinochchi district in June 2016 with the stocking of 100,000 post larvae $\left(P L_{15}\right)$ with the aim of improving livelihood of the fishermen by diversifying species in the reservoir. Return of $\underline{M}$. rosenbergii was found to be high with a recapture rate of $2.5 \%$ and return on investment of $360.6 \%$ within one year study period. Income from harvesting $\underline{M}$. rosenbergii improved the livelihood of fishers by increasing their income and interest in fisheries. Furthermore, the total yield increased with the increase in fishing effort during the study period. In addition, it was observed that male $\underline{M}$. rosenbergii had a significantly high mass and growth rate compared to females on average. The results of this study indicated that $\underline{M}$. rosenbergii stocking program was a cost effective and a successful venture in the development of livelihood of fishing community and fisheries production of the Puthumurippu reservoir.
\end{abstract}

Keywords: Macrobrachium rosenbergii, post larvae stocking, Puthumurippu medium perennial reservoir, recapture rate

\section{INTRODUCTION}

Sri Lanka has many reservoirs of varying nature and scale including perennial and seasonal reservoirs of large, medium and small sizes. While the primary purposes of these reservoirs are irrigation and generation of hydroelectricity, they are used for fisheries production too similar to other reservoirs in Asia (Amarasinghe et al., 2001). Making use of reservoirs for fisheries production in Asia in the past few decades have contributed both to increase the production of food protein and to increase the income in rural areas (Amarasinghe et al., 2001).

Majority of the fisheries production in Sri Lanka comes from marine fisheries with a share of $86 \%$ of total production. The rest $14 \%$ comes from inland fisheries and aquaculture. While commercial scale capture fisheries occur at major irrigation reservoirs (> $750 \mathrm{ha})$, small-scale fisheries occur in the medium scale reservoirs of 250-750 ha (Amarasinghe, 2014). Fisheries production in reservoirs in Sri Lanka emerged after the introduction of Oreochromis mossambicus to Sri Lankan freshwaters in 1952 (Athukorala \& Amarasinghe, 2010). Capture fisheries in inland waters, culture based fisheries and shrimp farming are part of inland fisheries in Sri Lanka. Culture based fisheries were incorporated as part of the national fisheries development plan of Sri Lanka in 1980 with free provisioning of fingerlings for

\footnotetext{
1 Department of Animal Science, Faculty of Agriculture, University of Peradeniya, Sri Lanka

* Corresponding author: r.rajeevan88@gmail.com
} 
stocking. However, the government support for aquaculture and inland fisheries was withdrawn in between 1990 and 1994 and revitalized in 1994 (Amarasinghe, 2014).

The Puthumurippu reservoir is a medium perennial reservoir located in Kilinochchi district, in the Northern Province of Sri Lanka (Figure 1). The extent of the reservoir is 377 ha at full supply level. The reservoir is mainly used for irrigation purpose where fisheries are a secondary activity. In addition, the reservoir is the water resource for the day-to-day activities of the community and for the animal husbandry. Since there is no industrial activities are taking place, the reservoir remains pristine. Compared to the other reservoirs found in Kilinochchi, production and income from fisheries from Puthumurippu medium perennial reservoir is generally low. In 2016, the average fish catch per fisherman per day from Iranamadu and Akkarayankulam reservoirs in Kilinochchi district were 6-10 kg and 4-7 $\mathrm{kg}$, respectively. For the same time period, fish catch per fisherman per day for Puthumurippu reservoir was $2-5 \mathrm{~kg}$ equivalent to the daily earning of Rs. 300-750 per day. Given the low income from fisheries, the fishermen had to involve in secondary livelihoods such as masonry and other wage earning activities for their living.

In an attempt to optimize the use of the reservoir and to improve the livelihood of the fishers, Macrobrachium rosenbergii was newly introduced to the Puthumurippu medium perennial reservoir in June 2016 through a stocking program. In this context, the present research was designed to assess the improvement in the livelihood of the fishermen engaged in fisheries in the Puthumurippu reservoir due to the introduction of $M$. rosenbergii. The study particularly concentrated on production variation of $M$. rosenbergii with variation in fishing effort, recapture rate of $M$. rosenbergii and growth variation between male and female $M$. rosenbergiiin the Puthumurippu reservoir.

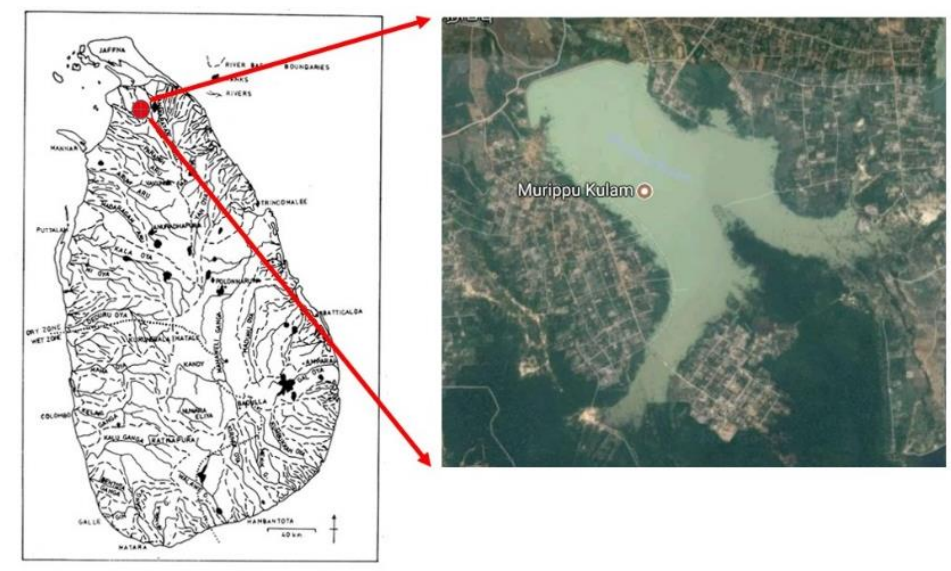

Figure1. Location of the Puthumurippu Reservoir

\section{MATERIALS AND METHODS}

The reservoir contained only one cohort of $M$. rosenbergii during the study period. The data related to catch, effort (number of active fishermen fishing at the Puthumuripu reservoir), stocking time, number of post larva (PL) and cost and revenues of $M$. rosenbergii fisheries were collected biweekly between June 2016 and July 2017. Gear used by the fishers was 2ply gill nets with mesh size ranging from a minimum of $8.89 \mathrm{~cm}$ to a maximum of $17.78 \mathrm{~cm}$. Local fishers assisted in fishing gear monitoring, though enforcement of regulations was 
mainly performed by National Aquaculture Development Authority (NAQDA). Catch data from the reservoir was estimated from the number, weight and sex of $M$. rosenbergii caught by fishers. $M$. rosenbergii landed by individuals or groups of fishers were identified, counted and their individual weights were recorded.

The appropriate timing for harvest (stocking to harvest) was estimated using the relationship between the month of stocking and growth of prawn as shown by the following equation:

$$
\mathrm{W}=\mathrm{a}+\mathrm{b} \mathrm{T} \text { (Sripatrprasite \& Lin, 2003) }
$$

Where, $\mathrm{W}=$ Weight of $M$. rosenbergii $(\mathrm{g})$

$$
\begin{aligned}
& \mathrm{T}=\text { Time of stocking } M \text {. rosenbergii (month) } \\
& \mathrm{b}=\text { Growth rate of } M . \text { rosenbergii } \\
& \mathrm{a}=\mathrm{Y} \text { intercept }
\end{aligned}
$$

Total revenue was calculated using the harvested weights by sex and farm-gate prices. The only cost associated with the stocking programme is the cost for stocking, which comprised of the total price of PLs and transport cost.

The relationship between the different parameters, such as yield, fishing effort, individual size of $M$. rosenbergii and production of $M$. rosenbergii were analysed and plotted using Minitab 17 statistical software and MS Excel (MS Office 2013) was used for recording the data.

\section{RESULTS AND DISCUSSION}

Prior to the introduction of $M$. rosenbergii the income of the fishermen from fishing at the Puthumurippu reservoir remained low (Rs 300-750 per day). Sometimes, the catch was as low as $1 \mathrm{~kg}$ with a meagre income of Rs150. After the introduction of M. rosenbergii, the fishermen are able to catch $2 \mathrm{~kg} \mathrm{M}$. Rosenbergii in a day for 6 days in a week. This gives them an average income of Rs 2000-3000 per day. Therefore, the introduction of $M$. rosenbergii increased the income of fishers by at 2.6 times or more. This has also reduced the involvement of these fishermen in secondary livelihoods and helped them to be actively engaged in fishing. For instance, they engaged in fisheries for 2-3 days a week before the introduction of $M$. rosenbergii and their engagement in fishing has increased to 6 days a week after introducing the $M$. rosenbergii. As a result of introducing $M$. rosenbergii, the number of active fishermen in Puthumurippu perennial reservoir has increased from 3 to 10 within the study period. As shown in Figure 2, the yield of the $M$. rosenbergii $(\mathrm{kg} / \mathrm{ha} / \mathrm{month})$ in Puthumurippu reservoir shows a linear relationship to fishing effort during the study period. Fishing effort (FE), defined by the total number of fishermen engaged in fishing activities in the reservoir, showed a linear relationship with yield $(\mathrm{TY}=\mathrm{kg} / \mathrm{ha} / \mathrm{month})$ and can be expressed as; $\mathrm{TY}=-0.0901+0.07943 \mathrm{FE}$

This relationship between the yield and fishing effort suggests that the Puthumurippu reservoir is subjected to sub-optimal fishing pressure and perhaps represents the ascending limb of a possible Schaefer-type curve. However, this does not mean that the fishing effort can be increased indefinitely but indicates that the relationship is valid for ranges in the yield and fishing effort observed during the study period.

The additional income from harvesting $M$. rosenbergii has brought other social benefits to the fishers, such as, refurbishment of houses, improve assets, increase the access to loans, and meeting the educational expenses of children. 


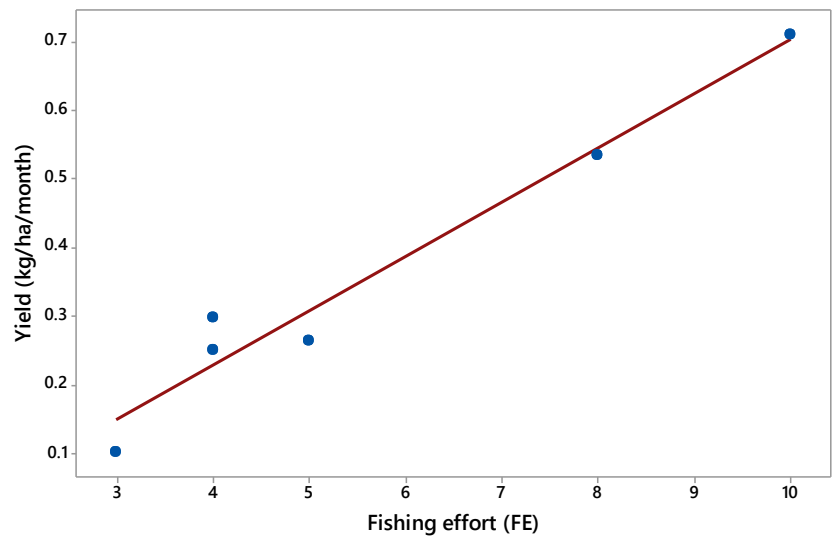

Figure 2. Relationship between the freshwater prawn (FWP) yield $(\mathrm{kg} / \mathrm{ha} / \mathrm{month})$ to fishing effort (No. of fishers) for Puthumurippu reservoir

Number of M. rosenbergii post-larvae stocked in June 2016 in the reservoir was 100,000. The total number $M$. rosenbergii harvested within a year was 2480 . Therefore, the recapture rate for the study period was $2.48 \%$. Mean weight of male $M$. rosenbergii caught in this reservoir was $389.0 \pm 5.9 \mathrm{~g}(\mathrm{n}=188)$ with a range of $230-540 \mathrm{~g}$ and the mean weight of females was $181.6 \pm 4.8 \mathrm{~g}(\mathrm{n}=51)$ with a range of $105-245 \mathrm{~g}$ (Figure 3). Compared to females, males showed high growth rate (Figure 3).

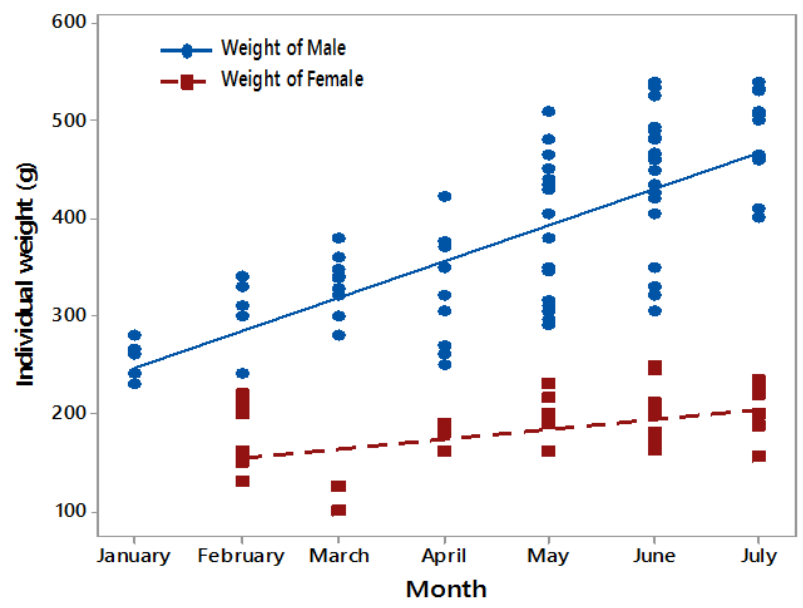

Figure 3. Weights of male and female $M$. rosenbergii in Puthumurippu reservoir (January-July 2017)

The relationship between the weight of male $M$. rosenbergii and time of stocking can be expressed as; $\mathrm{W}=-9.5+36.54 \mathrm{~T}\left(\mathrm{R}^{2}=47.11 \%\right)$. While that of female $M$. rosenbergii can be expressed as; $\mathrm{W}=74.1+9.97 \mathrm{~T}\left(\mathrm{R}^{2}=24.6 \%\right)$. Accordingly, a male $M$. rosenbergii was estimated to grow to reach $282.8 \mathrm{~g}$ in 8 months $(\mathrm{T}=8)$, with an average growth rate of 36.5 $\mathrm{g} / \mathrm{month}$. The female $M$. rosenbergii was estimated to grow up to $153.9 \mathrm{~g}$ in 8 months $(\mathrm{T}=8)$, with an average growth rate of $10.0 \mathrm{~g} /$ month. Despite the low $\mathrm{R}^{2}$ values observed for the model, nearly $50 \%$ of the variation in the weight of males is explained by the stocking 
period. Each reservoir is unique and hence, other factors such as feed and space availability, and the reservoir environment affect the growth of newly introduced species.

The total male M. rosenbergii catch was $702.1 \mathrm{~kg} / \mathrm{year}$ with a farm-gate value of Rs.842,496. Total catch of female $M$. rosenbergii was $112.3 \mathrm{~kg} / \mathrm{year}$ with a farm-gate value of Rs.78,631. The total yield was $814.4 \mathrm{~kg} /$ year (Figure 4) with a value of Rs.921,127. The total income from fisheries at the Puthumurippu reservoir was Rs.1,020,077 per year. Therefore, the revenue from $M$. rosenbergii contributes to $90.3 \%$ of fisheries income of the Puthumurippu reservoir. The reason for this significant contribution from the harvest of $M$. rosenbergii is also partly because of a change in fishing gear technique. Since the M. rosenbergii has a higher market value than other fish species, fishers are targeting it for harvest. Hence, they have modified their gear by adding more sinkers and few floaters to reach the bottom to catch $M$. rosenbergii. Since the Catla, Rohu and Tilapia inhabit the surface and column of the reservoir, the likelihood of catching them is low after the introduction of $M$. rosenbergii thus, become by catch. Economically, the cost of 100,000 PLs was Rs. 200,000 and the value of recaptured $M$. rosenbergii was Rs.921,127. Therefore, the return on investment was $360.6 \%$ per year with a $2.48 \%$ per year recapture rate.

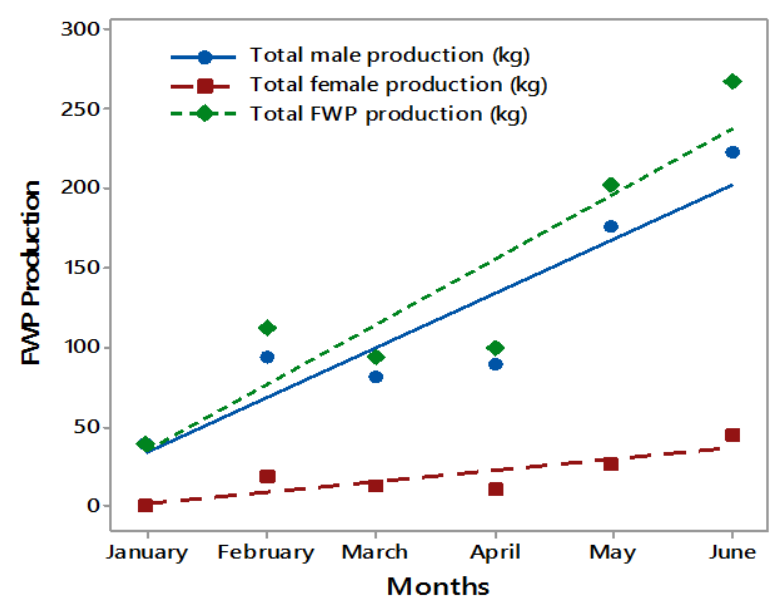

Figure 4. M. rosenbergii (FWP) production in Puthumurippu reservoir (January -June 2017)

The observed results of Puthumurippu medium perennial reservoir is comparable to the results of a study conducted for a year on the stocking program of Pak Mun Dam, which is a major reservoir of 4909 ha, in Thailand. While the average weight of the male $M$. rosenbergii in Puthumurippu was $389.0 \pm 5.9 \mathrm{~g}$, for the Pak Mun Dam reservoir average weight of the $M$. rosenbergii harvested during the one year study period was $236.5 \pm 102.1 \mathrm{~g}$. The M. rosenbergii harvest in the Pak Mun Dam reservoir contributed to $97 \%$ of the economic value of fisheries production of the reservoir, whereas for the Puthumurippu reservoir it contributed to $90.3 \%$ of revenue of fisheries production in the reservoir. Similarly, while the Pak Mun Dam had a yield of $2.4 \mathrm{~kg} / \mathrm{ha} / \mathrm{yr}$, for the study period Puthumurippu reservoir had a yield of $2.2 \mathrm{~kg} / \mathrm{ha} / \mathrm{yr}$. Comparing the revenue from the fresh water prawn caught, the Pak Mun Dam has higher revenue with 9.7 USD/ kg compared to 7.4 USD/ kg for Puthumurippu. On the other hand, Puthumurippu reservoir has a higher recapture rate of $2.48 \%$ per year compared to $1 \%$ per year recapture rate of Pak Mun Dam reservoir (Sripatrprasite \& Lin, 2003). As a whole, both studies highlighted stocking program could successfully contribute to the improvement of fisheries in reservoirs. Similar 
experiences of introduction of $M$. rosenbergii into reservoirs contributing to the development of fisheries in the reservoir can be found in the states of India, including Kerala (Laxmappa \& Krishna, 2015).

\section{CONCLUSIONS}

Introduction of $M$. rosenbergii is a successful endeavour improving the livelihood of fishers while increasing their interest in fisheries. Hence, culture fisheries are one of the best ways of utilising the reservoir while eradicating poverty. With the recapture rate of $2.48 \%$ per year with the return on investment of $360.6 \%$ per year, a remarkable improvement of fisheries production of the Puthumurippu Medium Perennial Reservoir occurred during the study period. Studies investigating on the factors affecting the growth of the $M$. rosenbergii and the yield of the reservoir over time need to be done further to better utilise the reservoir and to provide socio-economic benefits to poor fisher folks within the carrying capacity of the reservoir.

\section{ACKNOWLEGEMENT}

The authors would like to thank the fishermen who cooperated in conducting this study, the staffs of Kilinochchi NAQDA Regional Office and Divron Bioventures (Pvt.) Ltd., who shared data and lent logistic support.

\section{REFERENCES}

Amarasinghe, U. (2014). Fisheries resources in alleviation of hunger and malnutrition in Sri Lanka - accomplishment and challenges. SL J. Aqua. Sci. 18,1-15. DOI: http:// doi.org/10.4038/sljas.v18i0.7034

Amarasinghe, U., Duncan, A., Moreau, J., Schiemer, F., Simon, D. and Vijverberg, J. (2001). Promotion of sustainable capture fisheries and aquaculture in Asian reservoirs and lakes. Hydrobiologia. 458, 181-190. DOI: 10.1023/A:1013181522746

Athukorala, D. and Amarasinghe, U. (2010). Population dynamics of commercially important fish species in two reservoirs of the Walawe river basin, Sri Lanka. Asian Fish. Sci. 23, 71-90.

Laxmappa, B. and Krishna, S.M. (2015). Polyculture of the freshwater prawn Macrobrachium malcolmsonii (H.M. Edwards) in Koilsagar reservoir of Mahabubnagar district (TS), India. International J. Fish. \& Aqua. Stud. 2(4), 147-152.

Sripatrprasite, P. and Lin, C. (2003). Stocking and recapture of freshwater prawn (Macrobrachium rosenbergii De Man) in a run-of-river type dam (Pak Mun Dam) in Thailand. Asian Fish. Sci. 16, 167-174 